\title{
Total Glucosides of Paeony Inhibited Autophagy and Improved Acute Kidney Injury Induced by Ischemia-Reperfusion via the IncRNA TUG I/ miR-29a/PTEN Axis
}

\author{
Xiaoyan Chang' \\ Pei Zhang' \\ Xing-Xin $\mathrm{Xu}{ }^{\prime}$ \\ Bo Pang ${ }^{2}$
}

'Department of Nephropathy, The First Affiliated Hospital of Anhui Medical University, Hefei, Anhui, People's Republic of China; ${ }^{2}$ Department of Respiratory and Critical Care Medicine, The First Affiliated Hospital of Anhui Medical University, Hefei, Anhui, People's Republic of China
Correspondence: Bo Pang The First Affiliated Hospital of Anhui Medical University, No. 218 Jixi Road, Shushan District, Hefei, 230022, Anhui, People's Republic of China Tel +86 055I-62922913

Email Bopang1217@163.com
Objective: Total glucosides of paeony (TGP) has been proven to affect anti-inflammatory, immunomodulatory and hypoxia tolerance. This study investigates the effect of TGP on autophagy in acute kidney injury (AKI) induced by ischemia-reperfusion (I/R).

Methods: Rat model of AKI induced by $\mathrm{I} / \mathrm{R}$ was established. Rats were administered with TGP at different doses by oral gavage. The contents of BUN, creatinine, NGAL, Kim-1 and IL-18 were detected. The levels of inflammatory factors (TNF- $\alpha$, IL-1 $\beta$ and IL-6) and autophagy were measured. The expressions of lncRNA TUG1, miR-29a and PTEN were detected and their binding relationships were verified. I/R rat model with overexpressed TUG1 was established to explore the effect of TGP on kidney injury and autophagy. The hypoxia/reoxygenation (HR) model of HK-2 cells and the HR model of HK-2 cells overexpressing TUG1 and low-expressing PTEN were established.

Results: TGP decreased the contents of BUN, creatinine, NGAL, Kim-1 and IL-18, and reduced the levels of inflammatory factors. LncRNA TUG1 and PTEN were downregulated, and miR-29a was upregulated in kidney tissues. The binding relationships between lncRNA TUG1 and miR-29a, and miR-29a and PTEN were confirmed. TGP suppressed PTEN expression via the lncRNA TUG1/miR-29a axis. Overexpressing lncRNA TUG1 attenuated the protective effect of TGP on AKI and autophagy in HK-2 cells. TGP improved cell viability and inhibited the autophagy in HR model of HK-2 cells via lncRNA TUG1/miR29a/PTEN axis.

Conclusion: TGP inhibited autophagy and improved AKI induced by I/R via the lncRNA TUG1/miR-29a/PTEN axis.

Keywords: total glucosides of paeony, lncRNA TUG1/miR-29a/PTEN, ischemiareperfusion, acute kidney injury, autophagy

\section{Introduction}

Ischemia-reperfusion ( $/ / R$ ) injury refers to the cell and tissue damage caused by the recovery of blood flow after ischemia. ${ }^{1}$ The pathogenesis of I/R injury is complicated, and multiple factors are concerned with this pathological process such as ischemia, hypoxia, inflammation, autophagy, mitochondrial dysfunction and various signaling pathways. ${ }^{2}$ Acute kidney injury (AKI) resulted from I/R has been a thorny problem in clinic. ${ }^{3}$ It represents a frequent complication of surgical patients with a high mortality rate, which tends to develop into chronic kidney diseases and 
causes a great burden on social medical expenses. ${ }^{4}$ Considerable prevention and treatment strategies have been employed to reduce AKI; however, the outcomes of patients with AKI remain poor. ${ }^{5}$ Hence, further elucidating the pathogenesis of AKI and seeking the therapy of alleviating injury have become a hot issue.

Total glucosides of paeony (TGP) is a kind of glycoside mixture extracted from the root of Paeonia lactiflora. ${ }^{6}$ TGP bears anti-inflammatory and immunomodulatory properties, which has been extensively applied in human autoimmune diseases. ${ }^{7}$ In addition, TGP is reported to protect kidney from oxidative damage and prevent tubulointerstitial injury. ${ }^{8}$ A previous literature has shown that TGP has a protective effect on kidney injury in diabetic rats. ${ }^{9}$ However, relative little is known about the effect of TGP on AKI induced by $\mathrm{I} / \mathrm{R}$ in rats.

Emerging evidences have revealed that long non-coding RNAs (lncRNA) play a critical role in the occurrence and progression of kidney injury. ${ }^{5}$ For example, Sun et al have demonstrated that suppression of IncRNA CRNDE attenuates the kidney injury caused by sepsis, which is expected to be a clinical target of kidney injury. ${ }^{10}$ LncRNA taurine upregulated gene 1 (TUG1) has been demonstrated to be aberrantly expressed in human malignancies. ${ }^{11}$ For example, lncRNA TUG1 expression is promoted significantly in osteosarcoma cells, which may accelerate the course of osteosarcoma. ${ }^{12}$ What is more, it is reported that knockdown of lncRNA TUG1 can alleviate inflammation and apoptosis induced by $\mathrm{I} / \mathrm{R}{ }^{5}$ Shi et al have reported that inhibition of lncRNA TUG1 can reduce $\mathrm{I} / \mathrm{R}$ injury after acute myocardial infarction by inhibiting its target gene expression. ${ }^{13}$ Generally, traditional Chinese medicine plays a regulatory role in diseases by regulating the lncRNA expression. ${ }^{14-16}$ The correlation between TGP and lncRNA TUG1 remains unknown yet. Based on the previous findings, TGP may exert effect on I/ R-induced AKI in rats by modulating the lncRNA TUG1 expression. We hypothesize that TGP downregulates the expression of IncRNA TUG1 to regulate the downstream target genes, thus alleviating the I/R-induced AKI. Herein, we establish the rat model of AKI induced by I/R to investigate the specific mechanism of TGP and lncRNA TUG1 on kidney injury, which shall provide impetus for the determination of new therapeutic targets of AKI.

\section{Materials and Methods}

\section{Ethics Statement}

The study got the approval of the Ethical Committee of the First Affiliated Hospital of Anhui Medical University. All experimental procedures were implemented on the Ethical Guidelines for the Study of Experimental Pain in Conscious Animals.

\section{Animal Grouping and Establishment of Rat Model of AKI Induced by I/R}

Forty adult male Sprague-Dawley (SD) rats weighing 180-220 g were purchased from Hunan SJA Laboratory Animal Co., Ltd. [SYXK (Hunan) 2016-0002, Changsha, Hunan, China]. The rats were reared in standard animal room at $40-70 \%$ humidity and $18-22^{\circ} \mathrm{C}$. Food and water were provided ad libitum. The rats were maintained in a $12 \mathrm{~h}$ light/dark cycle. The rats were randomly assigned into 5 groups: sham group, IR group, IR + TGP-L group, IR + TGP-M group and IR + TGP-H group. The rat model of AKI was established by the previous studies. ${ }^{17,18}$ Briefly, the rats were anesthetized by intraperitoneal injection of pentobarbital sodium $(35 \mathrm{mg} / \mathrm{kg}$, Merck Serono, Geneva, Switzerland). A median abdominal incision was performed to expose bilateral renal pedicle vessels and separate bilateral renal arteries. The bilateral renal pedicle was clamped by a Schwartz microvessel clamp (18052-02, Fine Science Tools). The color of bilateral kidneys changed from bright red to pale, then to dark red. After $40 \mathrm{~min}$, the vascular clamp was released to restore perfusion, and the color of kidneys changed to bright red again. After the blood flow of the kidney was restored, the abdomen was closed layer by layer and the wound was sutured. During the operation, the body temperature of rats was maintained at $37^{\circ} \mathrm{C}$ on a constant temperature table. After the operation, $1 \mathrm{~mL}$ phosphate buffer saline (PBS) and $0.5 \mathrm{mg} / \mathrm{kg}$ buprenorphine (Sigma-Aldrich, Merck KGaA, Darmstadt, Germany) were injected via tail vein to maintain the postoperative fluid balance and relieve pain. After anesthesia, the rats were fed and drank freely. The rats in sham group were only treated by opening abdominal cavity; the rats in the IR + TGP-L group, IR + TGP-M group and IR + TGP$\mathrm{H}$ group were subjected to bilateral kidney $\mathrm{I} / \mathrm{R}$ operation to induced AKI. The rats in the sham group and the IR group were treated with $0.5 \%$ sodium carboxymethyl cellulose (30036328, Shanghai Sino Pharm Co., Ltd, Shanghai, China) by oral gavage for 3 consecutive days. The rats in the IR + TGP-L group, IR + TGP-M group and IR + TGP$\mathrm{H}$ group were administered with TGP suspension at different doses $(50 \mathrm{mg} / \mathrm{kg} / \mathrm{d}, 100 \mathrm{mg} / \mathrm{kg} / \mathrm{d}$ and $200 \mathrm{mg} / \mathrm{kg} / \mathrm{d})$ by oral gavage for 3 consecutive days (20171208, Liwah Pharmaceutical Co., Ltd, Ningbo, Jiangsu, China). ${ }^{19}$ The 
rats were anesthetized at 24,48 and $72 \mathrm{~h}$ after operation, and the tail vein blood was collected for biochemical index detection. A total of 7 rats died within 3 days after operation, which were excluded from the experiment. One rat in sham group died of intraoperative blood loss; three rats in the IR group and three rats in the IR + TGP-L group died of kidney ischemic necrosis; one rat in the IR + TGP-M group died of kidney ischemic necrosis; two rats in the IR + TGP-H group died of kidney artery embolism. At least 6 rats in each group survived 3 days after operation. On the 4th day after the operation, the rats were anesthetized by intraperitoneal injection of pentobarbital sodium $(50 \mathrm{mg} /$ $\mathrm{kg}$ ) and then euthanized by abdominal aorta bloodletting. The kidneys were removed. The left kidney was frozen in liquid nitrogen and stored at $-80^{\circ} \mathrm{C}$ for molecular biological detection. The right kidney was preserved in $4 \%$ paraformaldehyde solution for routine hematoxylin and eosin (HE) pathological section, immunohistochemistry, TUNEL staining and transmission electron microscope (TEM) analysis.

Based on the recovery of kidney injury in the above groups, the optimal dose of TGP (200 mg/kg) was determined. Adenovirus vector of overexpressed lncRNA TUG1 (ov-TUG1) and ov-NC $\left(1 \times 10^{8}\right.$ TU/mL, Gene Pharma, Shanghai, China) were constructed. Sixteen adult male Sprague-Dawley rats were randomly divided into IR + TGP + ov-TUG1 group and IR + TGP + ov-NC group according to body weight. The bilateral renal pedicle was also clamped with non-invasive artery clamp. During the clamping period, the bilateral kidneys of rats in the IR + TGP + ov-TUG1 group were injected with $1 \mathrm{~mL}$ ov-TUG1 virus solution at the speed of $100 \mu \mathrm{L} / \mathrm{min}$ through each renal artery. ${ }^{20}$ The rats in IR + TGP + ov-NC group were injected with $1.0 \mathrm{~mL}$ ov-NC virus solution. After clamping for $40 \mathrm{~min}$, the artery clamp was released to restore perfusion, and the subsequent abdominal closure, fluid infusion and analgesia were the same as those in the IR group. The rats in the two groups were administered TGP (200 mg/kg) by oral gavage for 3 consecutive days. Four rats died within 3 days after operation, and were excluded from the experiment. Two rat in the IR + TGP + ov-TUG1 group died of renal artery embolism, and two rats in the IR + TGP + ov-NC group died of kidney ischemic necrosis. Six rats in each group survived 3 days after operation. Blood and kidney samples of rats were collected according to the above methods for index detection.

\section{Detection of Serum Creatinine (Scr) and Urea Nitrogen (BUN)}

The blood was centrifuged at $4{ }^{\circ} \mathrm{C}$ for $10 \mathrm{~min}$. The contents of BUN and Scr were measured using Scr colorimetric assay (KitSarcosine oxidase method) (E-BC-K188-M, Elabscience Biotechnology Co., Ltd, Wuhan, China) on LABOSPECT0003 automatic blood biochemical analyzer (Hitachi, Tokyo, Japan).

\section{Enzyme-Linked Immunosorbent Assay (ELISA)}

The ground left kidney tissues were homogenized in $0.05 \%$ Tween 20 solution (Freemore, Beijing, China). After ultrasonic treatment for $20 \mathrm{~s}$, the homogenate was centrifuged at $4^{\circ} \mathrm{C}$ and $2500 \mathrm{~g}$ for $10 \mathrm{~min}$, or the collected rat blood was centrifuged at $4^{\circ} \mathrm{C}$ and $2500 \mathrm{~g}$ for $5 \mathrm{~min}$, and the supernatant was collected for ELISA. The levels of interleukin (IL)-18, kidney injury molecule 1 (Kim-1) and neutrophil gelatinaseassociated lipocalin (NGAL) in rat serum and the levels of tumor necrosis factor (TNF)- $\alpha$, IL-1 $\beta$ and IL-6 in rat kidney tissues were detected using ELISA kits (rat TNF- $\alpha$ ELISA kit, 48T-QS41721; rat IL-1ßELISA kit, 48T-QS41588; rat IL-6 ELISA kit, 48T-QS41731; rat IL-18 ELISA kit, 48TQS41735; rat Kim-1 ELISA kit, 48T-QS41858; rat NGAL ELISA kit, 48T-QS41812; all purchased from Beijing Gersion Bio-Technology Co., Ltd, Beijing, China).

\section{HE Staining}

The kidney tissue sections fixed by $4 \%$ paraformaldehyde were dehydrated with ethanol of gradient concentrations, cleared using xylene and embedded in paraffin. Next, the tissues were sliced at $4 \mu \mathrm{m}$, deparaffinized and dehydrated. Then, the sections were stained using the HE staining kit (Solarbio, Beijing, China). Afterwards, the tissue sections were dehydrated with ethanol of gradient concentrations, cleared using xylene, and sealed with neutral gum. The degree of tissue injury was observed under CX31 biomicroscope at $\times 200$ magnification (Olympus Optical Co., Ltd, Tokyo, Japan). The following criteria were used for tubular injury score: 0 , no obvious injury; 1 , mild injury, epithelial cell swelling and lumen expansion; 2 , severe injury, flattening of renal tubular epithelium, loss of nuclear staining and obstruction of lumen; 3, damaged renal tubular epithelial cells, cell abscission, nuclear staining disappearing, and a large number of tubules. Ten visual fields were randomly selected from each section for pathological evaluation. Blind 
method was used for random analysis: the readers did not know the grouping of rats.

\section{Terminal Deoxynucleotidyl Transferase (TdT)-Mediated dUTP Nick End Labeling (TUNEL) Staining}

The kidney tissue sections $(4 \mu \mathrm{m})$ were stained in line with the instructions of TUNEL kit (C1098, Beyotime Biotechnology Co., Ltd, Shanghai, China). Five fields were randomly determined from each section. The ratio of the number of TUNEL-positive cells to the total cells in each field was calculated, and the average value was taken as the rate of TUNEL-positive cells.

\section{Cell Culture and Grouping}

HK-2 cells were obtained from Cell Resource Center of Shanghai Institutes for Biological Sciences, the Chinese Academy of Sciences (Shanghai, China). Cells were cultured in Dulbecco's modified Eagle's medium (DMEM) containing $10 \%$ fetal bovine serum (FBS) and $1 \%$ penicillin-streptomycin at $37^{\circ} \mathrm{C}$ and $5 \% \mathrm{CO}_{2}$. Cells were subcultured when reaching $80 \%$ confluence. Different concentrations of TGP $(5,10,20,40,50,100$ and 200 $\mu \mathrm{g} / \mathrm{mL}$ ) were added to the culture medium for $48 \mathrm{~h}$-treatment. The appropriate IC50 concentration of TGP was determined according to the results of 3-(4,5-dimethylthiazol-2-yl)-2,5-diphenyltetrazolium bromide (MTT) assay. The hypoxia/reoxygenation $(\mathrm{H} / \mathrm{R})$ model in vitro was established by the following steps: ${ }^{21}$ HK-2 cells were cultured in the medium free of glucose and serum under the conditions of $1 \% \mathrm{O}_{2}, 5 \% \mathrm{CO}_{2}$ and $94 \% \mathrm{~N}_{2}$ for 24 h. Thereafter, the cells were cultured in the DMEM containing $10 \%$ FBS under the conditions of $21 \% \mathrm{O}_{2}, 5 \% \mathrm{CO}_{2}$ and $74 \% \mathrm{~N}_{2}$ for $12 \mathrm{~h}$.

HK-2 cells were assigned into Blank group (HK-2 cells grown under normal conditions), $\mathrm{H} / \mathrm{R}$ group (HK-2 cells were subjected to $\mathrm{H} / \mathrm{R}$ treatment), and $\mathrm{H} / \mathrm{R}+\mathrm{TGP}$ group (HK-2 cells were subjected to $\mathrm{H} / \mathrm{R}$ treatment and cultured with $50 \mu \mathrm{g} / \mathrm{mL}$ TGP for $48 \mathrm{~h}$ ). Some cells in H/R + TGP group were transfected with adenovirus vector or miRNA mimic or siRNA $12 \mathrm{~h}$ before $\mathrm{H} / \mathrm{R}$ treatment, and the other operations were the same as those in the $\mathrm{H} / \mathrm{R}+\mathrm{TGP}$ group.

\section{Cell Transfection}

The adenovirus vector of TUG1 overexpression (ovTUG1), adenovirus empty vector (ov-NC) $\left(1 \times 10^{8} \mathrm{Tu} /\right.$
$\mathrm{mL}$ ), small interfering RNA (siRNA) of PTEN (si-PTEN) and its empty control (si-NC) were purchased from Gene Pharma (Shanghai, China). The above vectors or RNAs were transfected into HK-2 cells using Lipofectamine ${ }^{\mathrm{TM}}$ 3000 (Invitrogen, Carlsbad, CA, USA).

\section{MTT Assay}

HK-2 cells were treated with TGP $(5,10,20,40,50,100$, and $200 \mu \mathrm{g} / \mathrm{mL}$ ) for $48 \mathrm{~h}$. Then, the original medium was removed and $100 \mu \mathrm{L}$ medium containing MTT $(5 \mathrm{mg} / \mathrm{mL})$ was added for $4 \mathrm{~h}$-incubation. Afterwards, the medium was removed and dimethyl sulphoxide solution was added to make the precipitate fully dissolved. The optical density (OD) of each well was measured at a wavelength of $570 \mathrm{~nm}$.

\section{TEM Analysis}

The fresh kidney was washed with $0.9 \%$ sodium chloride injection. The samples $(1 \mathrm{~mm})$ were put in $2.5 \%$ glutaraldehyde at $4{ }^{\circ} \mathrm{C}$ and fixed with $1 \%$ osmic acid solution. Then, the samples were dehydrated with ethanol of gradient concentrations and embedded in paraffin. Next, the samples were sliced at $70 \mathrm{~nm}$ and stained with uranyl acetate and lead citrate. Autophagosome and autolysosome were observed under the TEM (JEM, Tokyo, Japan).

HK-2 cells were collected and then fixed with $2.5 \%$ paraformaldehyde and $1.5 \%$ osmic acid solution. Next, the cells were dehydrated with ethanol of gradient concentrations, embedded in paraffin, sliced at $70 \mathrm{~nm}$ and stained with uranyl acetate and lead citrate. Autophagosome and autolysosome were observed under the TEM (JEM).

\section{Autophagy Flux Detection}

HK-2 cells in the blank, HR, HR + TGP, HR + TGP-ov$\mathrm{NC}$ and HR + TGP-ov-TUG1 group were transfected with GFP-RFP-LC3 adenovirus (Hanbio, Shanghai, China), respectively. The multiplicity of infection (MOI) was $20 .^{22}$ Then, the cells were stained with DAPI solution (Sangon, Shanghai, China) at room temperature for 5 min. TGP intervention $(50 \mu \mathrm{g} / \mathrm{mL})$ was performed in each group after $12 \mathrm{~h}$ of transfection. Autophagy flux was detected $48 \mathrm{~h}$ later. The cells were observed under confocal microscope (Olympus). Image $\mathrm{J}$ software was used for image analysis and processing, and the number of GFP and RFP fluorescent dots was counted. In the Merge image, autophagosomes were represented by yellow dots and autolysosomes were represented by red dots. Fluorescence intensity was expressed as the total number 
of dots divided by the number of nuclei in each microscope field.

\section{Dual-Luciferase Reporter Gene Assay}

The binding sites of lncRNA TUG1 and miR-29a, and miR-29a and PTEN were predicted by StarBase v2.0 (http://starbase.sysu.edu.cn/index.php). The binding and mutant sequences of H19 containing miR-29a and PTEN were amplified and then cloned to the pmirGLO luciferase vector (Promega, Madison, WI, USA). Wild-type (WT) plasmid (TUG1-WT/PTEN-WT) and mutant-type plasmid (TUG1-MUT/PTEN-MUT) were constructed. Then, the constructed vectors and miR-29a mimic (Gene Pharma) or mimic NC were co-transfected into HEK293T cells (Shanghai Institute of Cellular Biology of Chinese Academy of Sciences, Shanghai, China). Luciferase activities were detected using the dual-luciferase reporter assay system (Promega) after $48 \mathrm{~h}$ of transfection.

\section{RNA Pull-Down Assay}

The potential binding relationship between IncRNA TUG1 and miR-29a was detected using RNA pull-down assay. miR-29a-biotin, miR-29a-MUT-biotin and NC-biotin were transfected into HK-2 cells. After $24 \mathrm{~h}$ of culture, the cells and streptavidin magnetic beads were incubated in the lysis buffer (Ambion, Austin, Texas, USA) for $2 \mathrm{~h}$, followed by purifying the binding RNA. ${ }^{23}$ Reverse transcription quantitative polymerase chain reaction (RT-qPCR) was used to detect the abundance of lncRNA TUG1.

\section{Western Blot Analysis}

The kidney tissues ground in liquid nitrogen and HK-2 cells were lysed in the radio-immunoprecipitation assay (RIPA) buffer (Beyotime). The concentration of proteins extracted from tissues and cells was tested using the bicinchoninic acid assay kit (Beyotime). Then, $30 \mu \mathrm{g}$ total protein of each well was separated on SDS-polyacrylamide gel electrophoresis (12\% or $6 \%$ separating gel) and transferred onto polyvinylidene difluoride membranes. The membranes were blocked with $5 \%$ bovine serum albumin (BSA) and cultured with the primary antibodies: rabbit anti-LC3 (ab192890, 1:1000; LC3I, $16 \mathrm{KDa}, \mathrm{LC} 3 \mathrm{II}, 14 \mathrm{KDa}$ ), rabbit anti-p62 (ab109012, 1:1000, $62 \mathrm{KDa}$ ), rabbit anti-PTEN (ab267787, 1:1000, 54KDa) and rabbit anti-glyceraldehyde-3-phosphate dehydrogenase (GAPDH) (ab181602, 1:10,000, $36 \mathrm{KDa}$ ). Afterwards, the membranes were cultured with the secondary antibody goat anti-rabbit immunoglobulin $\mathrm{G}$ (IgG) (ZB5301, 1:5000, ZSGB-Bio Co., Ltd, Beijing, China). The gray value of the target band was analyzed by Image-Pro Plus 6.0 software (Media Cybernetics, Inc., Rockville, $\mathrm{MD}$, USA).

\section{RT-qPCR}

Total RNA was extracted from the kidney tissues or HK-2 cells based on the instructions of a TRIzol reagent (Invitrogen). The PrimeScript RT reagent kit (Takara, Dalian, China) was employed to reversely transcribe total RNA into cDNA. qPCR was performed using $\mathrm{SYBR}^{\circledR}$ Premix Ex Taq ${ }^{\mathrm{TM}}$ II (Takara) on the ABI 7900 HT fast PCR real-time system (Applied Biosystems, Foster city, CA, USA). The relative expression of miR and mRNAs was calculated by $2^{-\Delta \Delta C t}$ method, with U6 and GAPDH acting as the internal reference. Primer sequences are illustrated in Table 1.

\section{Statistical Analysis}

SPSS 21.0 (IBM Corp., Armonk, NY, USA) was utilized for data analysis. Kolmogorov-Smirnov test showed that the data were in normal distribution and expressed as mean \pm standard deviation. The one-way analysis of variance (ANOVA) was applied for comparisons among multigroups. Tukey's multiple comparison test was applied for the post hoc test after ANOVA. We used G*Power 3.0.10 for post-hoc power analysis, and the relevant results are shown in Table $\mathrm{S} 1$. The $p$ value was obtained from a two-tailed test, and $p<0.05$ meant statistical difference.

\section{Results}

\section{TGP Improved AKI Induced by I/R}

Traditional Chinese medicine improved AKI by reducing inflammatory reaction, programmed cell death, necrosis

Table I Primer Sequence for RT-qPCR

\begin{tabular}{|l|l|}
\hline Gene & Sequence (5'-3') \\
\hline LncRNA TUGI & $\begin{array}{l}\text { F: CAAGAAACAGCAACACCAGAAG } \\
\text { R: TAAGGTCCCCATTCAAGTCAGT }\end{array}$ \\
\hline miR29a & $\begin{array}{l}\text { F: CTGCTGATCATGGGCCTCCT } \\
\text { R: CTCCACAGGCTCGGGTTGTT }\end{array}$ \\
\hline U6 & $\begin{array}{l}\text { F: TTCTTGGGTAGTTTGCAGTT } \\
\text { R: TTCTTGGGTAGTTTGCAGTT }\end{array}$ \\
\hline PTEN & $\begin{array}{l}\text { F: ATACCAGGACCAGAGGAAACC } \\
\text { R: TTGTCATTATCCGCACGCTC }\end{array}$ \\
\hline GAPDH & $\begin{array}{l}\text { F: ACAGCAACAGGGTGG TGGAC } \\
\text { R: TTTGAGGGTGCAGCGAACTT }\end{array}$ \\
\hline
\end{tabular}


and reactive oxygen species. ${ }^{24}$ As the main active component of Radix Paeoniae Alba, TGP played a vital role in antiinflammation, analgesia, antioxidation, protection of liver and kidney injury and immune regulation. ${ }^{24-26}$ Hence, this study investigated the protective effect of different doses of TGP on AKI induced by $I / R$ in rats. The experimental process is shown in Figure 1A. Continuous monitoring of kidney injury markers in serum showed that the levels of Scr and BUN were increased after $\mathrm{I} / \mathrm{R}$ operation, reaching the peak at $48 \mathrm{~h}$ (Figure 1B, all $p<0.05$ ); the levels of NGAL, Kim-1, IL-18 in serum were notably increased, reaching the peak at $24 \mathrm{~h}$ (Figure 1C, all $p<0.05$ ); the levels of inflammatory markers TNF- $\alpha$, IL- $1 \beta$ and IL- 6 were significantly elevated (Figure 1D, all $p<0.05$ ). HE staining showed that the rats in the sham group showed normal kidney tissues, intact structure of glomerulus and renal tubules without obvious inflammatory cell infiltration, while rats in the IR group showed disorderly destroyed renal tubules, dilated lumen, and exfoliated brush border of epithelial cells with inflammatory cells infiltrated (Figure $1 \mathrm{E}$, all $p<0.05$ ). These results suggested that the rat model of AKI induced by $\mathrm{I} / \mathrm{R}$ was successfully established.

After administration of different doses of TGP by oral gavage, the levels of BUN and Scr of rats in the IR + TGP
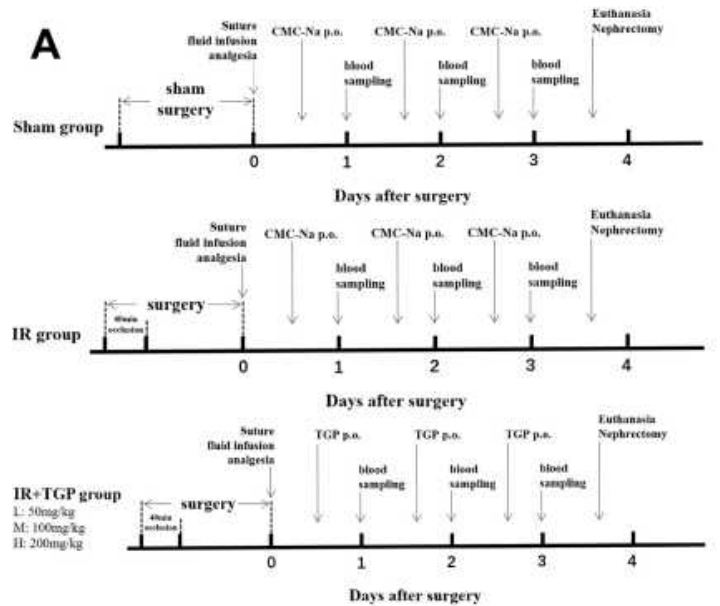

D
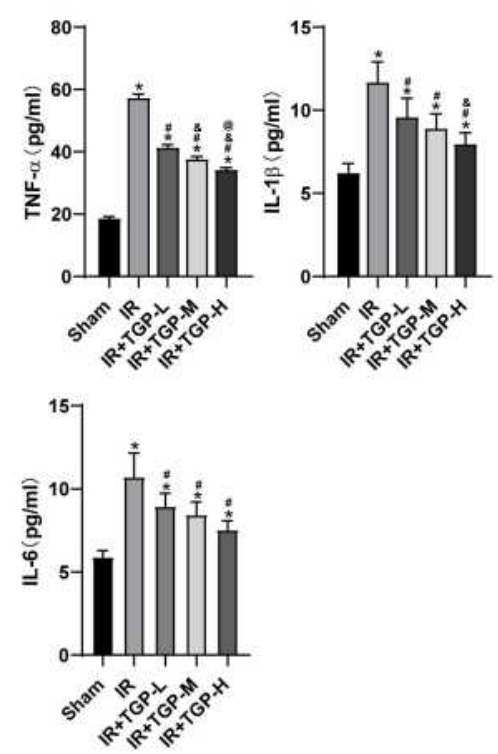

B
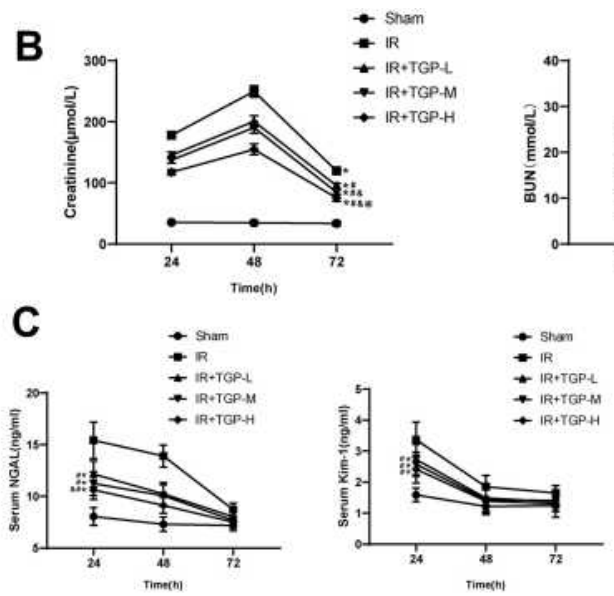

E
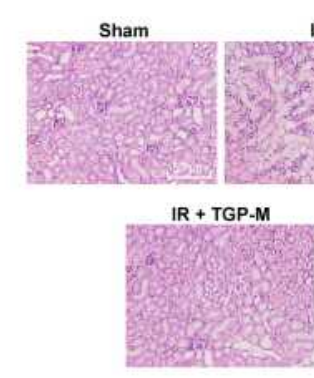

IR

IR + TGP-L
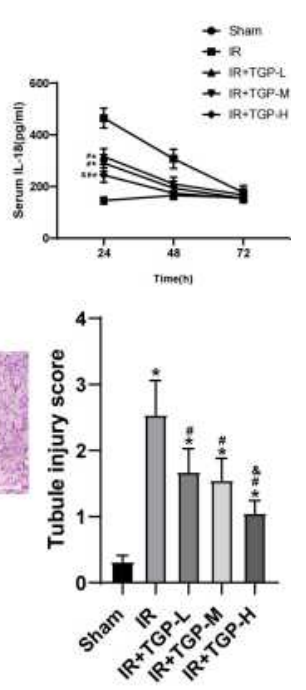

F

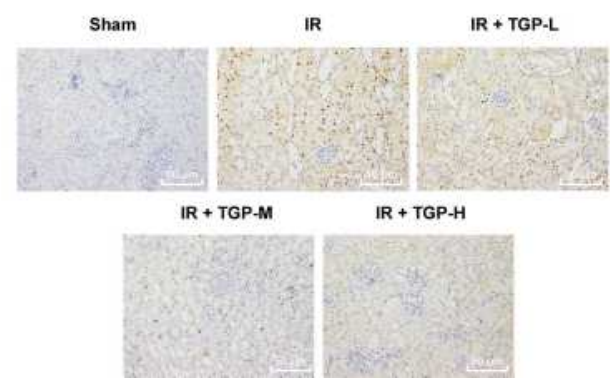

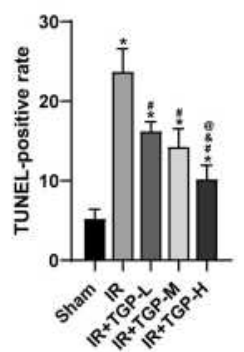

Figure I TGP improved rat AKI induced by I/R. (A) Schematic diagram of animal experimental time line of each group; (B) the contents of BUN and Scr of rats in each group were measured at 24, 48 and $72 \mathrm{~h}$ after kidney I/R operation; (C) the levels of NGAL, Kim-I and IL-I 8 of rat serum in each group were measured using ELISA at 24,48 and 72 $\mathrm{h}$ after kidney I/R operation; (D) the levels of TNF- $\alpha$, IL- I $\beta$ and IL- 6 of rat kidney tissues in each group were measured using ELISA at $72 \mathrm{~h}$ after kidney I/R operation; (E) HE staining and tubular injury score of rats in each group at $72 \mathrm{~h}$ after kidney I/R operation; (F) TUNEL staining of kidney tissues of rats in each group at $72 \mathrm{~h}$ after kidney l/R operation, and the positive cell rate was calculated. $N=6$. Data were expressed as mean \pm standard deviation and analyzed using one-way ANOVA, followed by Tukey's multiple comparison test, ${ }^{*} p<0.05$ vs the sham group; ${ }^{*} p<0.05$ vs the IR group; ${ }^{\&} p<0.05$ vs the IR + TGP-L group; ${ }^{Q_{p}}<0.05$ vs the IR + TGP-M group. 
groups were significantly lower than those in the IR group, showing a dose-dependent manner (Figure 1B, all $p<0.05$ ). Moreover, after administration of TGP, the levels of NGAL, Kim-1, IL-18 in serum were decreased (Figure 1C, all $p<$ 0.05 ) and the levels of TNF- $\alpha$, IL-1 $\beta$ and IL- 6 in kidney tissues were reduced (Figure 1D, all $p<0.05$ ). HE staining showed that the renal tubule injury of rats in the IR + TGP groups was notably reduced, but a small amount of tubular dilation and inflammatory cell infiltration were still observed (Figure 1E, all $p<0.05$ ). Compared with the rats in the sham group, those in the IR group showed increased number of TUNEL-positive cells, while compared with the IR group, the IR + TGP groups showed significantly decreased TUNEL-positive cells, showing a dose-dependent manner (Figure $1 \mathrm{~F}$, all $p<0.05$ ). These results indicated that different doses of TGP intervention significantly improved AKI induced by $\mathrm{I} / \mathrm{R}$. Rats in the IR + TGP-H group showed more obvious improvement of AKI.

\section{TGP Attenuated Autophagy in Rats with AKI Induced by I/R}

Autophagy was activated after kidney I/R, and excessive autophagy caused kidney tissue injury. ${ }^{27}$ To study the repairing mechanism of TGP on rat kidney injury induced by $I / R$, we further analyzed the effect of TGP on autophagy after I/R.
We found that the recovery of kidney injury of rats in the IR + TGP-H (200 mg/kg) group was more obvious than that in the IR + TGP-L and IR + TGP-M groups. Therefore, we further studied the effect of TGP-H on autophagy in rats. The results of transmission electron microscopy showed that autophagosome and autolysosome were observed in the sham group, IR group and IR + TGP-H group. Compared with that in the sham group, the number of autophagosome in rats in the IR group and IR + TGP-H group increased; compared with that in the IR group, the number of autophagosome and autolysosome in rats in the IR + TGP-H group decreased (Figure 2A). Compared with the rats in the sham group, those in the IR group and IR + TGP-H group showed significantly decreased expression of p62 and promoted ratio of LC3II/LC3I. The rats in the IR + TGP-H group showed an increased expression of p62 and a decreased expression of LC3II than the rats in the IR group (Figure 2B, all $p<0.01$ ).

\section{TGP Affected IncRNA TUG I and miR-29a Expression and Inhibited PTEN Expression}

Studies had shown that downregulation of lncRNA TUG1 and PTEN protected kidney from I/R injury. ${ }^{5,28}$ The binding sites of lncRNA TUG1 and miR-29a, and miR-29a and
A

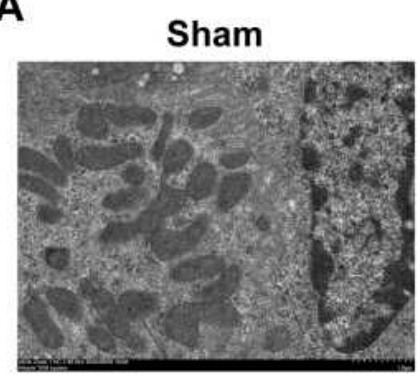

IR

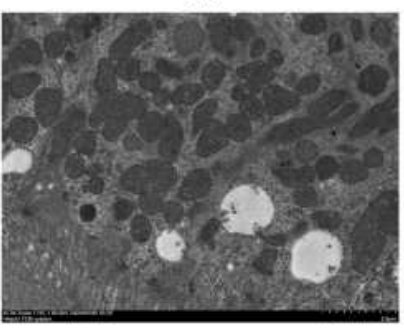

IR + TGP-H

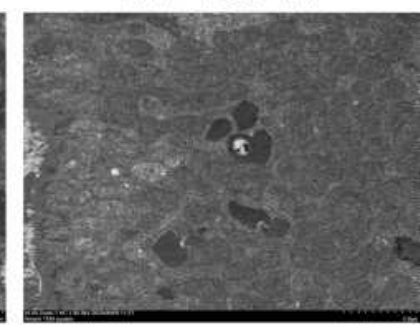

B
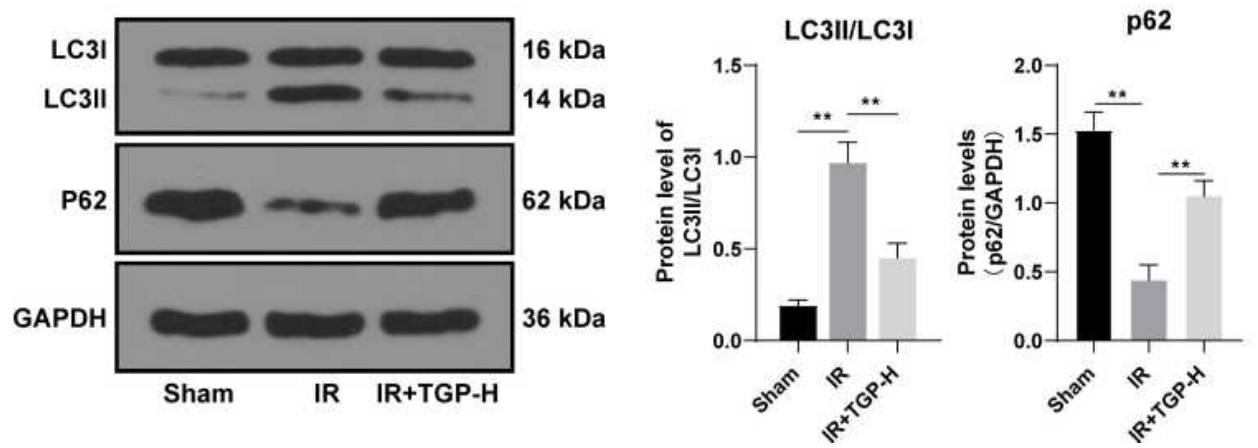

Figure 2 TGP attenuated autophagy in rats with AKI induced by I/R. (A) Autophagosome and autolysosome of rats in the sham group, IR group and IR + TGP-H group were detected using TEM assay; (B) the levels of p62 and LC3II/LC3I were detected using Western blot. $N=6$. Data were expressed as mean \pm standard deviation. Data in panel B were analyzed using one-way ANOVA, followed by Tukey's multiple comparison test, $*^{*} p<0.01$. 
PTEN were predicted by Targetscan and confirmed using dual-luciferase reporter gene assay (Figure $3 \mathrm{~A}, p<0.01$ ). Furthermore, RNA pull-down assay verified the binding relationship between miR-29a and lncRNA TUG1 (Figure $3 \mathrm{~B}, p<0.01)$. Therefore, we further studied the effect of TGP on the expression of IncRNA TUG1, miR-29a and PTEN in kidney after I/R. The rats in the IR group had an increased lncRNA TUG1 expression and PTEN expression and a decreased miR-29a compared with those in the sham group; the rats in the IR + TGP-H group showed an increased miR-29a expression and decreased lncRNA TUG1 and PTEN expression compared with those in the IR group (Figure 3C, all $p<0.01$ ). Western blot analysis also confirmed that PTEN level in kidney tissues was notably downregulated after TGP intervention (Figure 3D, $p<0.01$ ).

\section{Overexpression of IncRNA TUG I}

\section{Attenuated the Protective Effect of TGP on AKI Induced by I/R}

To further verify that TGP regulated autophagy after $\mathrm{I} / \mathrm{R}$ via the IncRNA TUG1/miR-29a/PTEN, we infected bilateral kidneys of rats with adenovirus to construct TUG1 overexpression rats. After I/R treatment, the rats were administered TGP by oral gavage for 3 days. Then, the effect of overexpression of lncRNA TUG1 on kidney injury and autophagy were analyzed. The operation processes are shown in Figure 4A. RT-qPCR confirmed that IncRNA TUG1 expression in the IR + ov-TUG1 + TGP-H group was significantly increased, indicating the successful transfection; moreover, miR-29a expression in the IR + ov-TUG1 + TGP-H group was decreased, indicating that lncRNA TUG1 could regulate miR-29a expression (Figure 4F, all $p<0.01)$. Compared with the IR + ov-NC + TGP-H group, the IR + ov-TUG1 + TGP-H group showed elevated Scr and BUN, increased NGAL, Kim-1 and IL-18 in serum and enhanced TNF- $\alpha$, IL-1 $\beta$ and IL-6 in kidney tissues; HE staining demonstrated that the IR + ov-TUG1 + TGP-H group had more severe damages to renal tubular structure, lumen expansion, epithelial brush border shedding and inflammatory cell infiltration (Figure 4B-E, all $p<0.01$ ). The rats in the IR + ov-TUG1 + TGP-H group showed upregulated LC3II/LCI ratio and PTEN protein level, and downregulated p62 level compared with those in the IR + ov$\mathrm{NC}+\mathrm{TGP}-\mathrm{H}$ group (Figure $4 \mathrm{G}$ and $\mathrm{H}$, all $p<0.01$ ), suggesting that overexpression of lncRNA TUG1 could reverse the inhibitory effect of TGP on PTEN expression and autophagy.

\section{TGP Inhibited Autophagy in HK-2 Cell Model of $H / R$}

HK-2 cells were treated with different concentrations of TGP $(5,10,20,40,50,100$ and $200 \mu \mathrm{g} / \mathrm{mL})$. After $48 \mathrm{~h}$ of incubation, the cell viability was evaluated. The results of MTT assay revealed that $100 \mu \mathrm{g} / \mathrm{mL}$ and $200 \mu \mathrm{g} / \mathrm{mL}$ TGP exerted obvious inhibitory effect on HK-2 cells, while $50 \mu \mathrm{g} / \mathrm{mL}$ TGP had no obvious inhibitory effect on $\mathrm{HK}-2$ cells (Figure 5A, all $p<$ $0.01)$. Therefore, $50 \mu \mathrm{g} / \mathrm{mL}$ TGP was selected as the intervention dose for HK-2 cells in the following study. We established HR model of HK-2 cells to study the effect of TGP on autophagy in HK-2 cells. Autophagosome and autolysosome were observed in the blank group, HR group and HR + TGP group. Compared with the blank group, the number of autophagosome in the HR group and HR + TGP group increased; compared with the HR group, the number of autophagosome and autolysosome in the HR + TGP group decreased (Figure $5 \mathrm{~B})$. The cells in the HR group showed an increased LC3II expression and a decreased p62 expression compared with those in the blank group; the cells in the HR + TGP group had a promoted p62 expression and a reduced ratio of LC3II/ LC3I than those in the HR group (Figure 5C, all $p<0.01$ ). The detection of autophagy flux further confirmed that TGP inhibited the occurrence of autophagy (Figure 5D, all $p<0.01$ ). The expressions of lncRNA TUG1, miR-29 and PTEN were detected using RT-qPCR. Compared with those in the HR group, the expressions of IncRNA TUG1 and PTEN decreased, while miR-29a expression increased significantly in the HR + TGP group (Figure 5E, all $p<0.01$ ).

\section{Overexpression of IncRNA TUG I Attenuated the Effect of TGP on Autophagy in HK-2 Cells}

To further determine whether TGP affected autophagy via the lncRNA TUG1/miR-29a/PTEN axis, we transfected HK2 cells with lncRNA TUG1 overexpression, and then performed HR and TGP treatment. MTT assay showed that compared with that in the HR + ov-NC + TGP group, the viability of cells in the HR + ov-TUG1 + TGP group was significantly decreased (Figure 6A, $p<0.01$ ). Compared with the HR + ov-NC + TGP group, the HR + ov-TUG1 + TGP showed increased lncRNA TUG1 expression, decreased miR-29a expression (Figure 6B-C, all $p<0.01$ ), and reduced PTEN protein level (Figure 6D, all $p<0.01$ ); additionally, HK-2 cells in the HR + ov-TUG1 + TGP group showed increased LC3II/LC3I expression and decreased p62 expression compared with those in the HR + ov-NC + TGP group 
A

TUG1 : 5' ggcuucuUUUUACAUGGUGCUg 3' miR-29a : 3' auuggcuAAAGUCUACCACGAu 5' TUG1-MUT: 5' ggcuucuCGCUGCCGAAGACUg 3' PTEN : 5' uuuuuuaaagCAUAUUGGUGCUa 3' miR-29a : 3' auuggcuaaaGUCU-ACCACGAu 5' PTEN-MUT: 5' uuuuuuaaagGCUGUCUACTCUa 3'

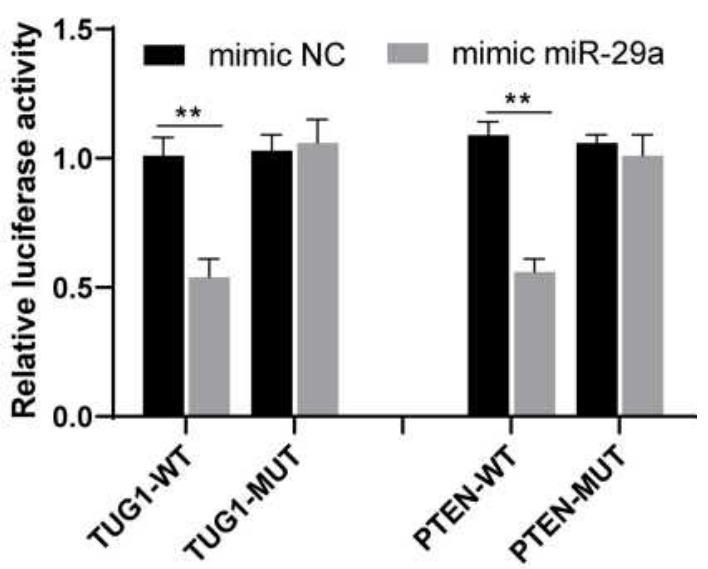

B

C
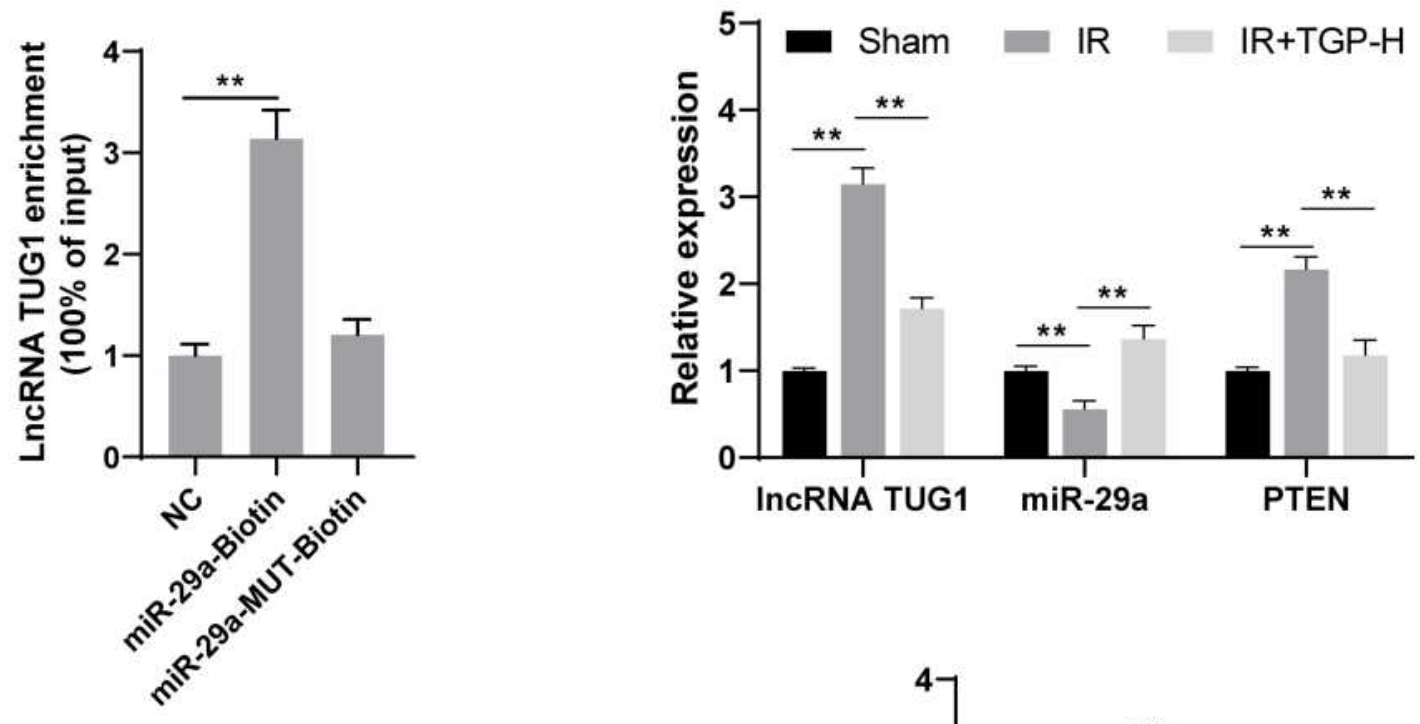

D
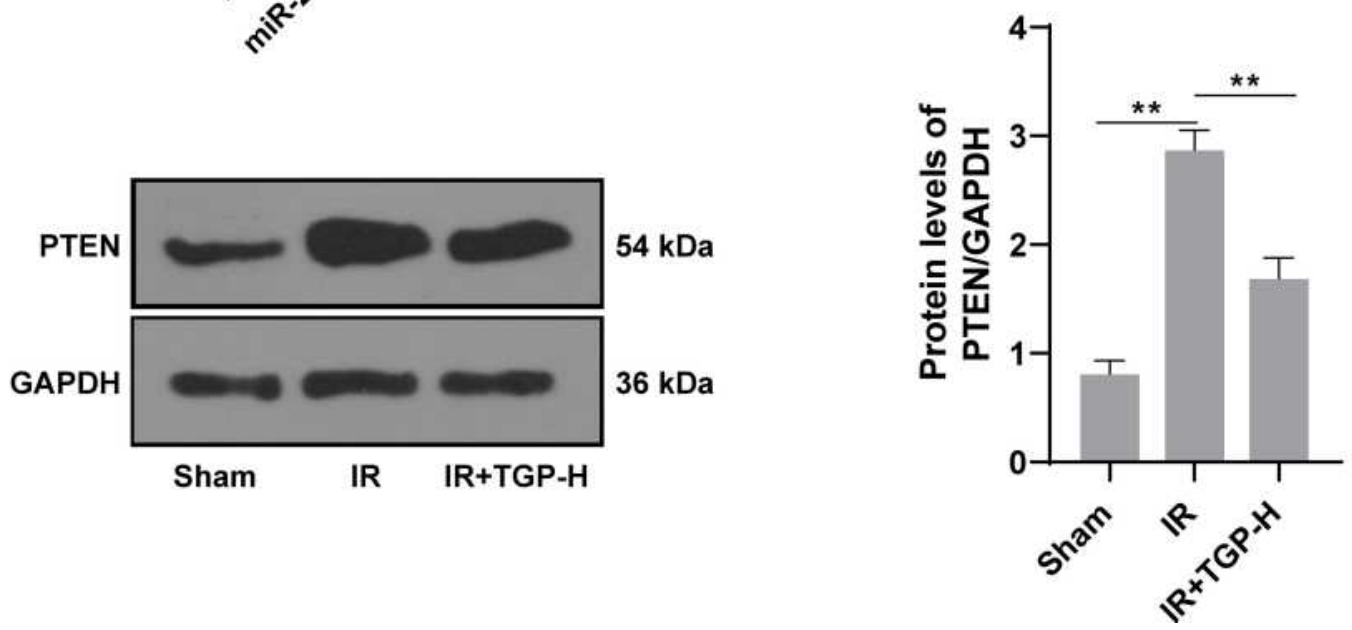

Figure 3 TGP inhibited PTEN expression via the IncRNA TUGI/miR-29a axis. (A) The targeting relationship among IncRNA TUGI/miR-29a/PTEN was confirmed using dual-luciferase reporter gene assay; (B) the targeting relationship between IncRNA TUGI and miR-29a was verified using RNA pull-down assay; (C) the expressions of IncRNA TUGI, miR-29a and PTEN were detected using RT-qPCR, and the target gene PTEN of IncRNA TUG I was verified using Western blot, N = 6; (D) the level of PTEN of rats in the sham, IR and IR + TGP-H group was detected using Western blot, $\mathrm{N}=6$. The cell experiment was repeated 3 times. Data were expressed as mean \pm standard, and analyzed using one-way ANOVA, followed by Tukey's multiple comparison test, $*^{*} p<0.0$ I. 
A

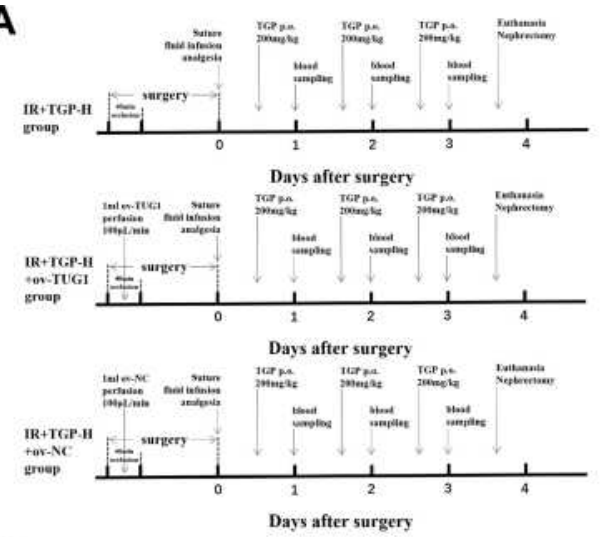

D

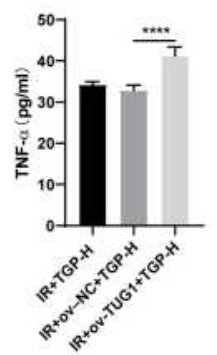

$\mathbf{F}$
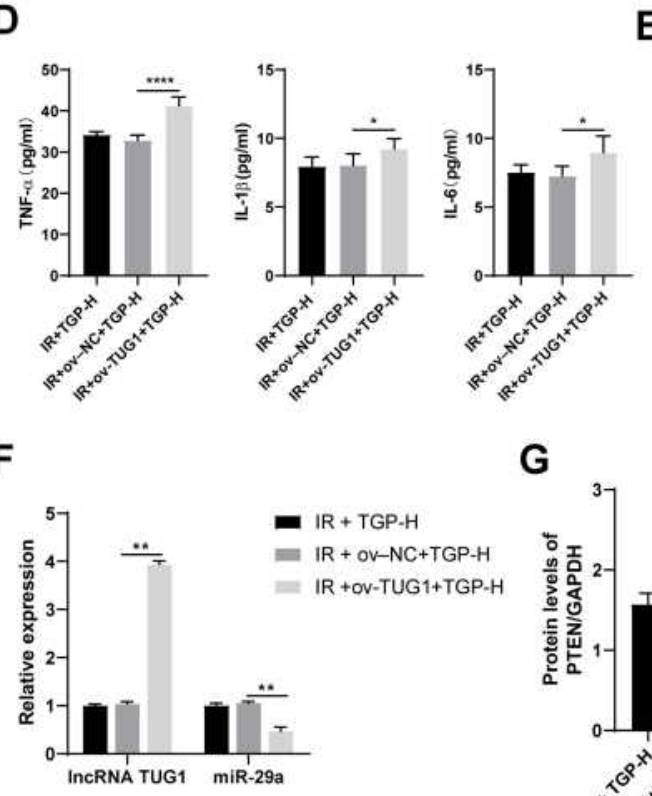

G
B

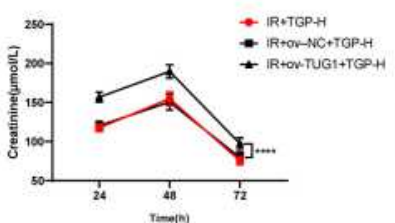

C
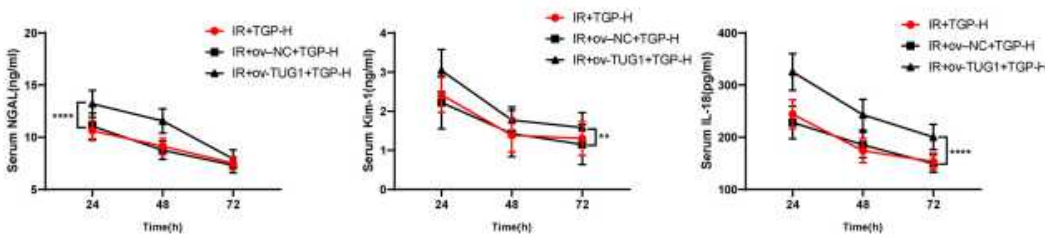

E

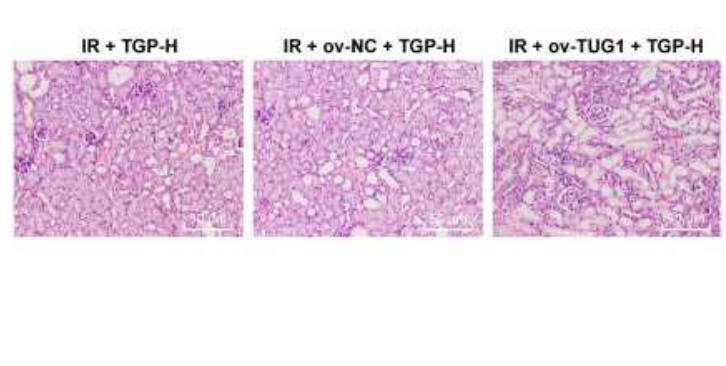

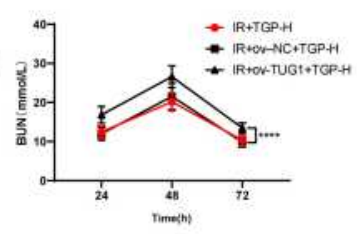

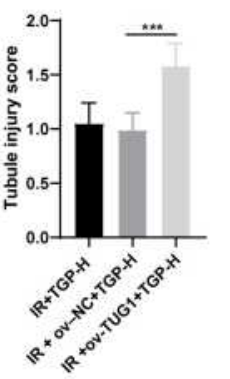

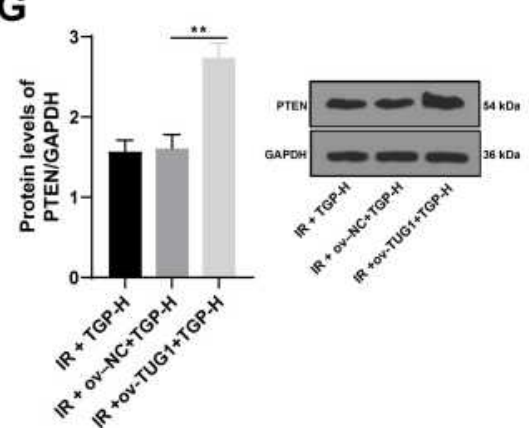

H

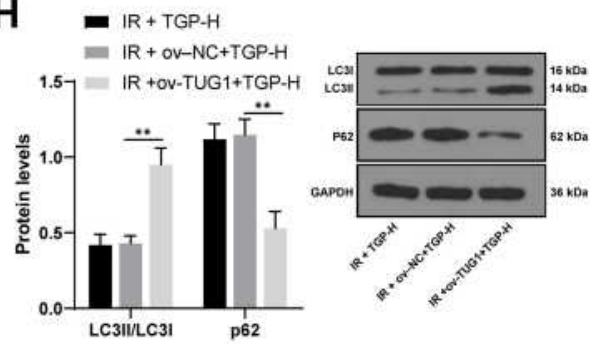

Figure 4 Overexpression of IncRNA TUGI attenuated the protective effect of TGP on AKI induced by I/R. (A) Schematic diagram of animal experimental time line of each group; (B) the contents of BUN and Scr of rat serum in each group were measured at 24, 48 and $72 \mathrm{~h}$ after kidney I/R operation; (C) the levels of NGAL, Kim-I and IL-I8 of rat serum in each group were measured using ELISA at 24,48 and $72 \mathrm{~h}$ after kidney I/R operation; (D) the levels of TNF- $\alpha$, IL-I $\beta$ and IL- 6 of rat kidney tissues in each group were measured using ELISA at $72 \mathrm{~h}$ after kidney l/R operation; (E) HE staining and tubular injury score; (F) The expressions of IncRNA TUGI and miR-29a of rats in each group were detected using RT-qPCR; (G) PTEN level of rats in each group was detected using Western blot; $(\mathbf{H})$ the levels of p62 and LC3 were detected using Western blot. $\mathrm{N}=6$. Data were expressed as mean \pm standard deviation, and analyzed using one-way ANOVA, followed by Tukey's multiple comparison test, ${ }^{*} p<0.05$, $* * p<0.0 \mathrm{I}$, $* * * p<0.001$, **** $p<0.000$ I.

(Figure 6E, all $p<0.01$ ). The occurrence of autophagy was further detected by GFP-RFP-LC3 transfection. It was found that overexpression of IncRNA TUG1 attenuated the inhibitory effect of TGP on autophagy (Figure 6F, $p<0.01$ ). HK-2 cells were transfected with si-PTEN to establish the cell model lowly expressing PTEN, and found that PTEN knockdown could increase cell viability and reduce autophagy. Next, we performed functional rescue experiment, HK-2 cells were co-transfected with ov-TUG1 and si-PTEN, and found that si-PTEN could reverse the effect of IncRNA TUG1 overexpression on cell viability and autophagy. It was confirmed that TGP affected autophagy via the lncRNA TUG1/miR-29a/PTEN axis.

\section{Discussion}

$\mathrm{I} / \mathrm{R}$ represented the major cause of AKI and currently there were no effective therapeutic approaches available. ${ }^{29}$ TGP was reported to bear anti-inflammatory and antioxidative effects, which could ameliorate kidney injury in diabetic mice. ${ }^{30}$ However, the effect of TGP on rats with AKI induced by $\mathrm{I} / \mathrm{R}$ remained to be clarified. We were the first to demonstrate that TGP inhibited autophagy and 
A

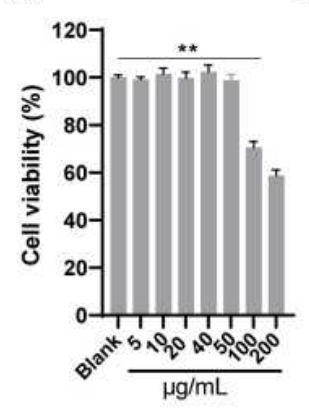

B

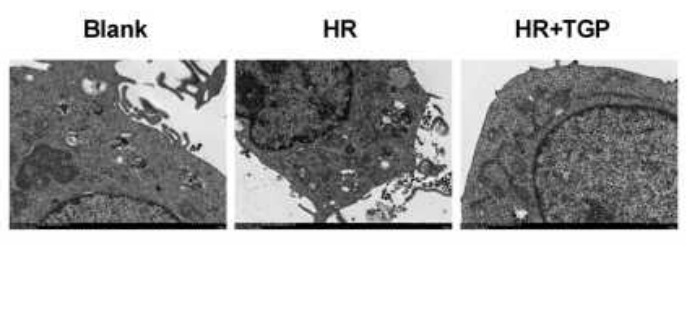

C

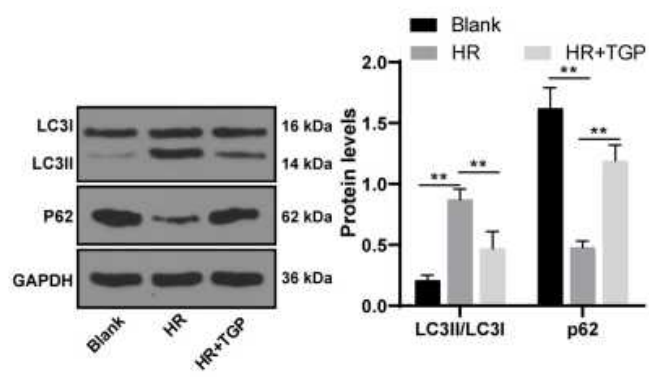

D

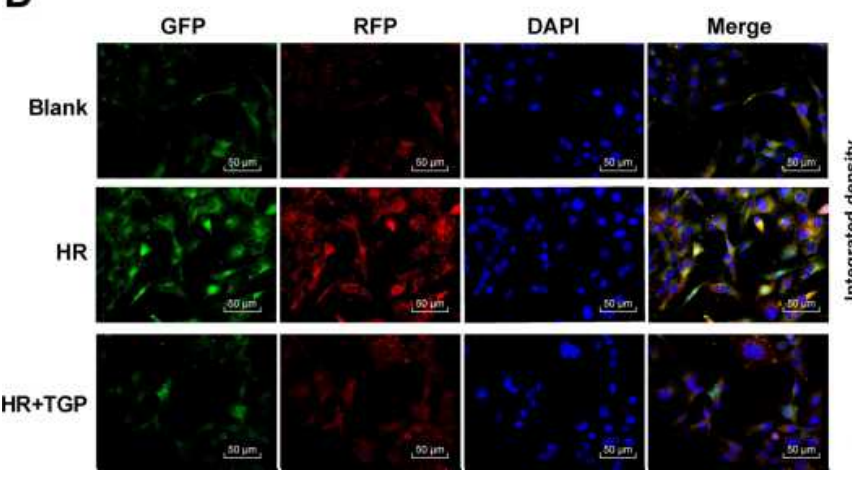

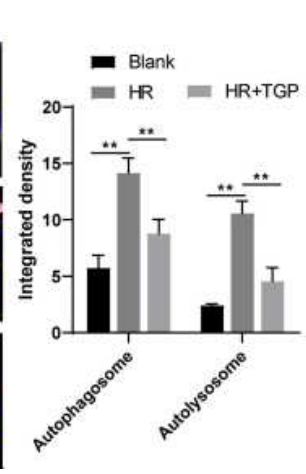

E

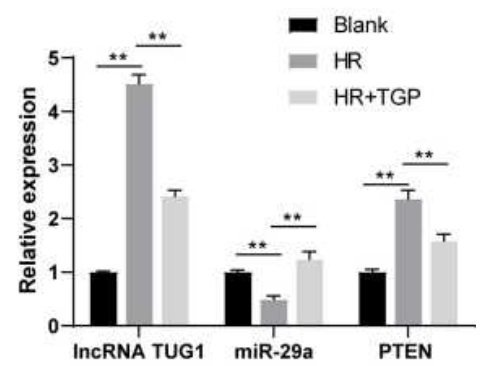

Figure 5 TGP inhibited autophagy in HR model of HK-2 cells. (A) The effects of different concentrations of TGP (5, 10, 20, 40, 50, I00, 200 $\mu$ g/mL) on HK-2 cell viability; (B) autophagosome and autolysosome of cells in the blank, HR and HR + TGP-50 group were detected using transmission electron microscopy; (C) the levels of p62 and LC3II/LC3I of cells in the blank, HR and HR + TGP-50 group were detected using Western blot; (D) the autophagic flow of cells in the blank, HR and HR + TGP-50 group was detected using immunofluorescence; (E) the mRNA expressions of IncRNA TUGI, miR-29a and PTEN of cells in the blank, HR and HR + TGP-50 group were detected using RT-qPCR. The cell experiment was repeated 3 times. Data were expressed as mean \pm standard deviation, and analyzed using one-way ANOVA, followed by Tukey's multiple comparison test, $* * p<0.01$.

improved AKI induced by $\mathrm{I} / \mathrm{R}$ via the lncRNA TUG1/miR29a/PTEN axis.

We established the rat model of AKI induced by $\mathrm{I} / \mathrm{R}$ to explore the protective effect of TGP on kidney injury in rats. Elevated expression of BUN and Scr was the biochemical marker of kidney injury. ${ }^{31}$ NGAL, Kim-1 and IL-18 not only allow an early diagnosis of AKI, but also provide prognostic information. ${ }^{32}$ Previous literature had shown that inhibiting kidney I/R-induced inflammation and enhancing tubular cell proliferation might be promising ways to relieve AKI. ${ }^{33}$ In this study, the levels of markers of kidney injury (BUN and Scr), biomarkers in the diagnosis of AKI (NGAL, Kim-1 and IL-18) in rat serum, and inflammation markers (TNF- $\alpha$, IL$1 \beta$ and IL-6) in rat kidney tissues were measured to evaluate the effect of TGP on AKI. The results demonstrated that after TGP intervention, the levels BUN, Scr, NGAL, Kim-1, IL18 , TNF- $\alpha$, IL- $1 \beta$ and IL-6 were significantly decreased in the I/R rats. NGAL, Kim-1 and IL-18 are biomarkers for the diagnosis and quantification of AKI, which are more sensitive than Scr and BUN. ${ }^{34}$ In this study, Scr and BUN reached the peak at $48 \mathrm{~h}$ after I/R operation, while NGAL, Kim-1 and IL-18 all increased prior to Scr, indicating that these proteins are helpful for early diagnosis of AKI. Additionally, the renal tubule injury of rats in the IR + TGP group was also notably reduced, with less inflammatory cell infiltration and TUNELpositive cells. Li et al also reported that TGP mitigated cerebral $\mathrm{I} / \mathrm{R}$ injury in rats by inhibiting inflammation and apoptosis. $^{35}$ These findings indicated that TGP could improve AKI induced by I/R.

Accumulating studies had revealed the critical role of autophagy in AKI induced by $\mathrm{I} / \mathrm{R}$ or nephrotoxic agents. ${ }^{36-38}$ Whether autophagy was destructive or protective in AKI remained unclear. The formation of autophagosome is one of the key events in autophagy, in which the newly synthesized membrane expands through the endomembrane-derived liposomes to form a complete autophagosome. ${ }^{39} \mathrm{LC} 3$ protein is indispensable for autophagy formation, and the LC3 II/LC3I ratio is considered to be a marker of autophagy. ${ }^{40}$ Moreover, p62 is a typical autophagy receptor distributed in cells and is implicated in 

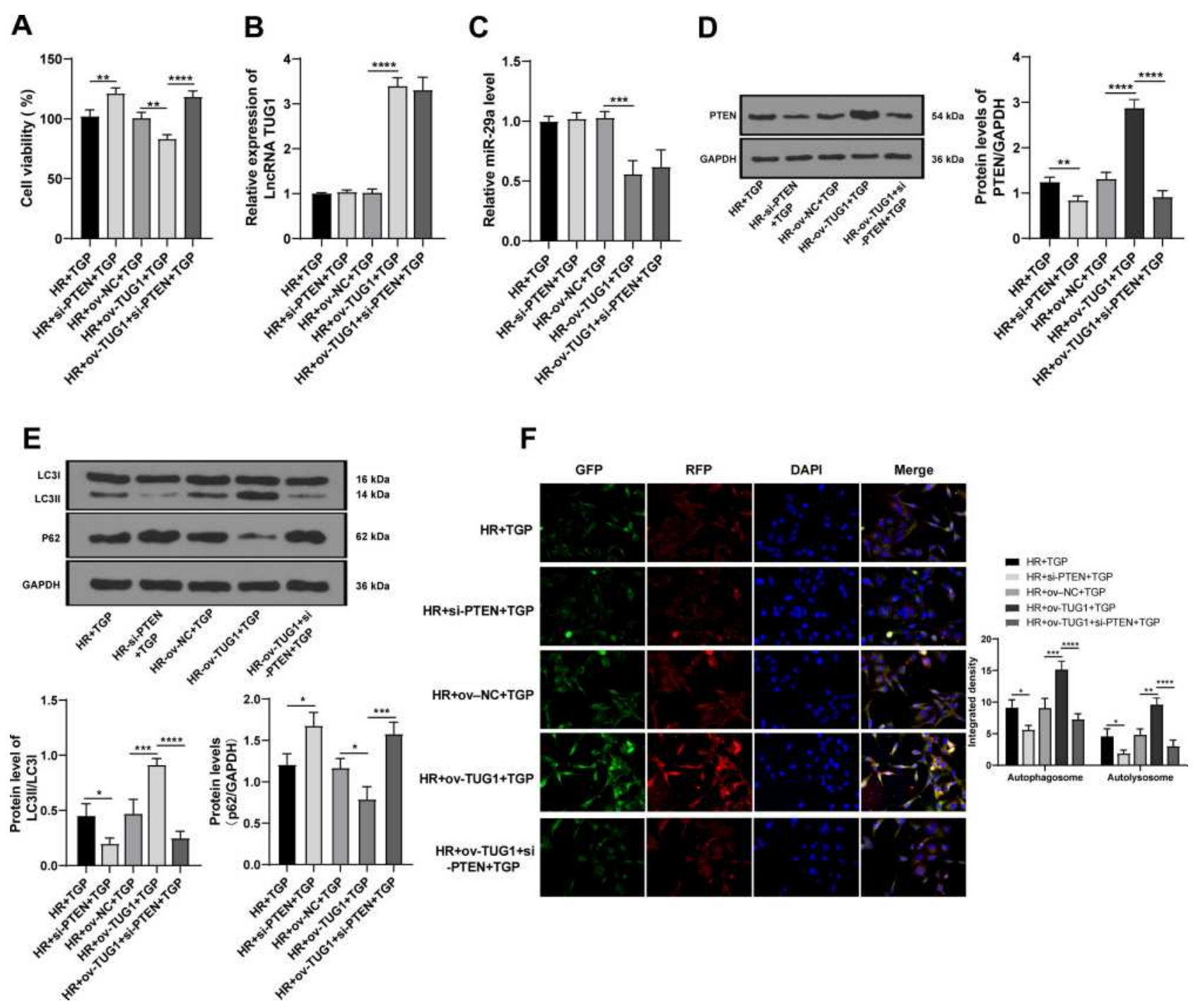

Figure 6 TGP attenuated autophagy in HR model of HK-2 cells via the IncRNA TUGI/miR-29a/PTEN axis. (A) The cell viability was detected using MTT assay; (B) the expression of IncRNA TUGI in each group of cells was detected using RT-qPCR; (C) the expression of miR-29a in each group of cells was detected using RT-qPCR; (D) the level of PTEN in each group of cells was detected using Western blot; (E) the levels of p62 and LC3II/LC3I in each group of cells were detected using Western blot; (F) the autophagic flow was detected using immunofluorescence. The cell experiment was repeated 3 times. Data were expressed as mean \pm standard deviation, and analyzed using one-way ANOVA, followed by Tukey's multiple comparison test, $*_{p}<0.05$, $* * p<0.01$, $* * * p<0.001$, $* * * * p<0.000$ I.

signal transduction pathways. ${ }^{41}$ This study revealed that the number of autophagosome and autolysosome in rats in the IR + TGP-H group decreased significantly as well as the reduced ratio of $\mathrm{LC} 3 \mathrm{II} / \mathrm{LC} 3 \mathrm{I}$ and promoted $\mathrm{p} 62$ expression. Excessive autophagy could cause widespread cell death and kidney tissue damages. ${ }^{27}$ In brief, TGP attenuated autophagy in rats with AKI induced by I/R. The results of in vitro experiments in HR-stimulated HK2 cells were generally in agreement with those in IRinduced rat model. It was indicated that TGP inhibited the autophagy in HK-2 cells, and overexpression of lncRNA TUG1 attenuated the effect of TGP on autophagy in HK-2 cells.
Emerging evidence had implied that lncRNAs were involved in the progression of renal diseases. ${ }^{42}$ It had been reported that IncRNA TUG1 contributed to the occurrence of chronic renal diseases. ${ }^{43}$ This study also found that lncRNA TUG1 was upregulated in rats with AKI. Mechanically, IncRNAs worked as endogenous miR sponges and then formed lncRNA-miR axis to modulate cell processes including apoptosis and autophagy. ${ }^{42}$ The binding relationship between miR-29a and lncRNA TUG1 was verified using RNA pull-down assay in this study. Diabetic mice with overexpressing miR-29a showed better renal tubular cell viability, indicating that the protective effect of miR-29a presented in 
the renal microenvironment. ${ }^{44}$ Thereafter, we focused on the downstream target gene regulated by lncRNA TUG1/ miR-29a. PTEN had critical influences on the pathogenesis of kidney injuring by regulating inflammation and apoptosis. ${ }^{45}$ For instance, Viñas et al exhibited that the protective effect of exosomes on kidney $\mathrm{I} / \mathrm{R}$ injury in mice was related to the decreased PTEN expression in kidney. ${ }^{46}$ The binding sites of IncRNA TUG1 and miR29a, and miR-29a and PTEN were confirmed using dualluciferase reporter gene assay. The rats in the IR + TGP$\mathrm{H}$ group showed an increased miR-29a expression and decreased lncRNA TUG1 and PTEN expression. TGP could inhibit PTEN expression via the lncRNA TUG1/ miR-29a axis. Then, we performed functional rescue experiments to analyze the effects of lncRNA TUG1 overexpression and PTEN silencing on autophagy, which further verified that TGP regulated autophagy via the lncRNA TUG1/miR-29a/PTEN axis.

To summarize, our study elaborated that TGP could alleviate AKI induced by I/R via the lncRNA TUG1/miR$29 \mathrm{a} / \mathrm{PTEN}$ axis. Our results provided essential evidence to further understand the protective mechanism of TGP in AKI induced by $\mathrm{I} / \mathrm{R}$. In the future, we shall carry out more prospective trials to refine our clinical guidance.

\section{Data Sharing Statement}

All the data generated or analyzed during this study are included in this published article.

\section{Funding}

This work was partially supported by the National Natural Science Foundation of China Youth Program (Grant No. 81700606). The funding body did not participate in the design of the study and collection, analysis, and interpretation of data and in writing the manuscript.

\section{Disclosure}

The authors declare that they have no competing interests.

\section{References}

1. Zuluaga Tamayo M, Choudat L, Aid-Launais R, et al. Astaxanthin complexes to attenuate muscle damage after in vivo femoral ischemia-reperfusion. Mar Drugs. 2019;17(6):6. doi:10.3390/ md17060354

2. Tang J, Zhuang S. Histone acetylation and DNA methylation in ischemia/reperfusion injury. Clin Sci (Lond). 2019;133(4):597-609. doi:10.1042/CS20180465

3. Yang K, Li WF, Yu JF, Yi C, Huang WF. Diosmetin protects against ischemia/reperfusion-induced acute kidney injury in mice. J Surg Res. 2017;214:69-78. doi:10.1016/j.jss.2017.02.067
4. Hobson C, Ruchi R, Bihorac A. Perioperative acute kidney injury: risk factors and predictive strategies. Crit Care Clin. 2017;33 (2):379-396. doi:10.1016/j.ccc.2016.12.008

5. Xu Y, Niu Y, Li H, Pan G. Downregulation of 1ncRNA TUG1 attenuates inflammation and apoptosis of renal tubular epithelial cell induced by ischemia-reperfusion by sponging miR-449b-5p via targeting HMGB1 and MMP2. Inflammation. 2020;43(4):1362-1374. doi:10.1007/s10753-020-01214-Z

6. Tu J, Guo Y, Hong W, et al. The regulatory effects of paeoniflorin and its derivative paeoniflorin-6'-O-benzene sulfonate CP-25 on inflammation and immune diseases. Front Pharmacol. 2019;10:57. doi:10.3389/fphar.2019.00057

7. Shen M, Men R, Fan X, et al. Total glucosides of paeony decreases apoptosis of hepatocytes and inhibits maturation of dendritic cells in autoimmune hepatitis. Biomed Pharmacother. 2020;124:109911. doi:10.1016/j.biopha.2020.109911

8. Lv M, Zhang M, Cheng Y, Zhang K, Hou C, Chen X. Renoprotective effects of total glucosides from paeony against nephrotoxicity induced by total alkaloids from semen strychni. Evid Based Complement Alternat Med. 2017;2017:8256278. doi:10.1155/2017/8256278

9. Zhang W, Zhao L, Su SQ, Xu XX, Wu YG. Total glucosides of paeony attenuate renal tubulointerstitial injury in STZ-induced diabetic rats: role of Toll-like receptor 2. J Pharmacol Sci. 2014;125 (1):59-67. doi:10.1254/jphs.13173FP

10. Sun BQ, Sui YD, Huang H, Zou XB, Chen SC, Yu ZK. Effect of IncRNA CRNDE on sepsis-related kidney injury through the TLR3/ NF-kappaB pathway. Eur Rev Med Pharmacol Sci. 2019;23 (23):10489-10497. doi:10.26355/eurrev_201912 19688

11. Zhao L, Sun H, Kong H, Chen Z, Chen B, Zhou M. The Lncrna-TUG 1/EZH2 Axis promotes pancreatic cancer cell proliferation, migration and emt phenotype formation through sponging mir-382. Cell Physiol Biochem. 2017;42(6):2145-2158. doi:10.1159/000479990

12. Sheng K, Li Y. LncRNA TUG1 promotes the development of osteosarcoma through RUNX2. Exp Ther Med. 2019;18(4):3002-3008. doi:10.3892/etm.2019.7880

13. Shi H, Dong Z, Gao H. LncRNA TUG1 protects against cardiomyocyte ischaemia reperfusion injury by inhibiting HMGB1. Artif Cells Nanomed Biotechnol. 2019;47(1):3511-3516. doi:10.1080/21691401.2018.1556214

14. Wei LB, Gao JR, Gao YC, Liu XC, Jiang H, Qin XJ. Effect of the traditional Chinese medicine Qi Teng Xiao Zhuo granules on chronic glomerulonephritis rats studied by using long noncoding RNAs expression profiling. Gene. 2020;728:144279. doi:10.1016/j. gene.2019.144279

15. Yu X, Tang W, Yang $\mathrm{Y}$, et al. Long noncoding RNA NKILA enhances the anti-cancer effects of baicalein in hepatocellular carcinoma via the regulation of NF-kappaB signaling. Chem Biol Interact. 2018;285:48-58. doi:10.1016/j.cbi.2018.02.027

16. Yu X, Yang Y, Li Y, et al. Baicalein inhibits cervical cancer progression via downregulating long noncoding RNA BDLNR and its downstream PI3K/Akt pathway. Int $J$ Biochem Cell Biol. 2018;94:107-118. doi:10.1016/j.biocel.2017.11.009

17. Sakai K, Nozaki Y, Murao Y, et al. Protective effect and mechanism of IL-10 on renal ischemia-reperfusion injury. Lab Invest. 2019;99 (5):671-683. doi:10.1038/s41374-018-0162-0

18. Tuuminen R, Jouppila A, Salvail D, et al. Dual antiplatelet and anticoagulant APAC prevents experimental ischemia-reperfusioninduced acute kidney injury. Clin Exp Nephrol. 2017;21 (3):436-445. doi:10.1007/s10157-016-1308-2

19. Wang K, Wu YG, Su J, Zhang JJ, Zhang P, Qi XM. Total glucosides of paeony regulates JAK2/STAT3 activation and macrophage proliferation in diabetic rat kidneys. Am J Chin Med. 2012;40(3):521-536. doi:10.1142/S0192415X12500401

20. Lu JX, Yan CY, Pu JX, Hou JQ, Yuan HX, Ping JG. [Gene transfer-induced human heme oxygenase-1 over-expression protects kidney from ischemia-reperfusion injury in rats]. Zhonghua Yi Xue Za Zhi. 2010;90(46):3300-3304. Chinese. 
21. Ye N, Xie D, Yang B, Li M. The mechanisms of the herbal components of CRSAS on HK-2 cells in a hypoxia/reoxygenation model based on network pharmacology. Evid Based Complement Alternat Med. 2020;2020:5352490. doi:10.1155/2020/5352490

22. Xie Y, Xiao J, Fu C, Zhang Z, Ye Z, Zhang X. Ischemic preconditioning promotes autophagy and alleviates renal ischemia/reperfusion injury. Biomed Res Int. 2018;2018:8353987. doi:10.1155/2018/ 8353987

23. Zhao CC, Jiao Y, Zhang YY, et al. Lnc SMAD5-AS1 as ceRNA inhibit proliferation of diffuse large B cell lymphoma via Wnt/beta-catenin pathway by sponging miR-135b-5p to elevate expression of APC. Cell Death Dis. 2019;10(4):252. doi:10.1038/s41419-0191479-3

24. Li HD, Meng XM, Huang C, Zhang L, Lv XW, Li J. Application of herbal traditional Chinese medicine in the treatment of acute kidney injury. Front Pharmacol. 2019;10:376. doi:10.3389/fphar.2019.00376

25. Su J, Zhang P, Zhang JJ, Qi XM, Wu YG, Shen JJ. Effects of total glucosides of paeony on oxidative stress in the kidney from diabetic rats. Phytomedicine. 2010;17(3-4):254-260. doi:10.1016/j. phymed.2009.07.005

26. Zhang L, Wei W. Anti-inflammatory and immunoregulatory effects of paeoniflorin and total glucosides of paeony. Pharmacol Ther. 2020;207:107452. doi:10.1016/j.pharmthera.2019.107452

27. Tan X, Zhu H, Tao Q, et al. FGF10 protects against renal ischemia/ reperfusion injury by regulating autophagy and inflammatory signaling. Front Genet. 2018;9:556. doi:10.3389/fgene.2018.00556

28. Zhou J, Zhong J, Lin S, et al. Inhibition of PTEN activity aggravates post renal fibrosis in mice with ischemia reperfusion-induced acute kidney injury. Cell Physiol Biochem. 2017;43(5):1841-1854. doi: $10.1159 / 000484070$

29. Liu H, Wang L, Weng X, et al. Inhibition of Brd4 alleviates renal ischemia/reperfusion injury-induced apoptosis and endoplasmic reticulum stress by blocking FoxO4-mediated oxidative stress. Redox Biol. 2019;24:101195. doi:10.1016/j.redox.2019.101195

30. Wu Y, Ren K, Liang C, et al. Renoprotective effect of total glucosides of paeony (TGP) and its mechanism in experimental diabetes. J Pharmacol Sci. 2009;109(1):78-87. doi:10.1254/jphs.08112FP

31. Niknahad H, Heidari R, Mohammadzadeh R, et al. Sulfasalazine induces mitochondrial dysfunction and renal injury. Ren Fail. 2017;39(1):745-753. doi:10.1080/0886022X.2017.1399908

32. Zdziechowska M, Gluba-Brzozka A, Poliwczak AR, et al. Serum NGAL, KIM-1, IL-18, L-FABP: new biomarkers in the diagnostics of acute kidney injury (AKI) following invasive cardiology procedures. Int Urol Nephrol. 2020;52(11):2135-2143. doi:10.1007/ s11255-020-02530-x

33. Qin C, Li M, Bai T, Yang K, Xu T, Tisp ZJ. 40 deficiency limits renal inflammation and promotes tubular cell proliferation in renal ischemia reperfusion injury. Exp Cell Res. 2018;371(1):255-261. doi:10.1016/j.yexcr.2018.08.019
34. Andreucci M, Faga T, Pisani A, Perticone M, Michael A. The ischemic/nephrotoxic acute kidney injury and the use of renal biomarkers in clinical practice. Eur J Intern Med. 2017;39:1-8. doi:10.1016/j.ejim.2016.12.001

35. Li LY, Ma L, Dong WL. Total glucosides of paeony (Paeonia lactiflora) alleviates blood-brain barrier disruption and cerebral ischemia/ reperfusion injury in rats via suppressing inflammation and apoptosis. Pharmazie. 2020;75(5):208-212. doi:10.1691/ph.2020.0324

36. Basile DP, Anderson MD, Sutton TA. Pathophysiology of acute kidney injury. Compr Physiol. 2012;2(2):1303-1353. doi:10.1002/ cphy.c110041

37. He L, Livingston MJ, Dong Z. Autophagy in acute kidney injury and repair. Nephron Clin Pract. 2014;127(1-4):56-60.

38. Mizushima N, Komatsu M. Autophagy: renovation of cells and tissues. Cell. 2011;147(4):728-741. doi:10.1016/j.cell.2011.10.026

39. Osawa T, Kotani T, Kawaoka T, et al. Atg2 mediates direct lipid transfer between membranes for autophagosome formation. Nat Struct Mol Biol. 2019;26(4):281-288. doi:10.1038/s41594-0190203-4

40. Gong L, He J, Sun X, Li L, Zhang X, Gan H. Activation of sirtuin1 protects against ischemia/reperfusion-induced acute kidney injury. Biomed Pharmacother. 2020;125:110021. doi:10.1016/j. biopha.2020.110021

41. Liu WJ, Ye L, Huang WF, et al. p62 links the autophagy pathway and the ubiqutin-proteasome system upon ubiquitinated protein degradation. Cell Mol Biol Lett. 2016;21(1):29. doi:10.1186/s11658016-0031-z

42. Tao Q, Tianyu W, Jiangqiao Z, et al. Expression analysis of long non-coding RNAs in a renal ischemia-reperfusion injury model. Acta Cir Bras. 2019;34(4):e201900403. doi:10.1590/s0102865020190040000003

43. Li SY, Susztak K. The long noncoding RNA Tug1 connects metabolic changes with kidney disease in podocytes. J Clin Invest. 2016;126 (11):4072-4075. doi:10.1172/JCI90828

44. Lin CL, Lee PH, Hsu YC, et al. MicroRNA-29a promotion of nephrin acetylation ameliorates hyperglycemia-induced podocyte dysfunction. $J$ Am Soc Nephrol. 2014;25(8):1698-1709. doi:10.1681/ASN.2013050527

45. Zhou J, Jia L, Hu Z, Wang Y. Pharmacological inhibition of PTEN aggravates acute kidney injury. Sci Rep. 2017;7(1):9503. doi:10.1038/s41598-017-10336-8

46. Vinas JL, Burger D, Zimpelmann J, et al. Transfer of microRNA-486$5 \mathrm{p}$ from human endothelial colony forming cell-derived exosomes reduces ischemic kidney injury. Kidney Int. 2016;90(6):1238-1250. doi:10.1016/j.kint.2016.07.015

\section{Publish your work in this journal}

Drug Design, Development and Therapy is an international, peerreviewed open-access journal that spans the spectrum of drug design and development through to clinical applications. Clinical outcomes, patient safety, and programs for the development and effective, safe, and sustained use of medicines are a feature of the journal, which has also been accepted for indexing on PubMed Central. The manuscript management system is completely online and includes a very quick and fair peer-review system, which is all easy to use. Visit http://www. dovepress.com/testimonials.php to read real quotes from published authors. 